\title{
Standardization of Optical Coherence Tomography Angiography Imaging Biomarkers in Diabetic Retinal Disease
}

\author{
Stela Vujosevic ${ }^{a}$ José Cunha-Vaz ${ }^{b, c}$ João Figueira ${ }^{b, c}$ Anat Löwenstein ${ }^{d}$ \\ Edoardo Midena ${ }^{e}$ Mariacristina Parravano ${ }^{f}$ Peter Henry Scanlon ${ }^{g}$ \\ Rafael Simó $^{h, i}$ Cristina Hernández ${ }^{h, i}$ Maria H. Madeira ${ }^{b, c}$ Inês P. Marques ${ }^{b, c, j}$ \\ António C.-V. Martinhob Ana R. Santos ${ }^{\mathrm{b}, \mathrm{j}}$ Olga Simó-Servat ${ }^{\mathrm{h}, \mathrm{i}}$ \\ Recivall P. Salongcay ${ }^{k, l}$ Dinah Zur ${ }^{d}$ Tunde Peto $^{k}$
}

${ }^{a}$ Eye Clinic, IRCCS MultiMedica, Milan, Italy; ${ }^{b}$ AIBILI-Association for Innovation and Biomedical Research on Light and Image, Coimbra, Portugal; 'Coimbra Institute for Clinical and Biomedical Research (iCBR), Faculty of Medicine, University of Coimbra, Coimbra, Portugal; ${ }^{\mathrm{d} O p h t h a l m o l o g y}$ Division, Tel Aviv Medical Center, affiliated to Sackler Faculty of Medicine, Tel Aviv University, Tel Aviv, Israel; 'Department of Neuroscience, University of Padua,

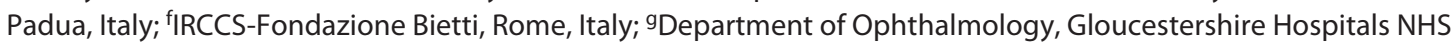
Foundation Trust, Cheltenham, UK; ' ${ }^{2}$ Diabetes and Metabolism Research Unit, Vall d'Hebron Research Institute, Barcelona, Spain; 'Centro de Investigación Biomédica en Red de Diabetes y Enfermedades Metabólicas Asociadas (CIBERDEM), Instituto de Salud Carlos III, Madrid, Spain; 'Department of Orthoptics, School of Health, Polytechnic of Porto, Porto, Portugal; ' $C e n t r e$ for Public Health, Queen's University Belfast, Belfast, UK; 'Eye and Vision Institute, The Medical City, Pasig, Philippines

\section{Keywords}

OCT angiography $\cdot$ Diabetic retinal disease $\cdot$ Imaging biomarkers $\cdot$ Standardization of terminology

\begin{abstract}
Optical coherence tomography Angiography (OCT-A) represents a revolution in the noninvasive evaluation of retinal and choroidal circulation especially in detecting early clinical signs of diabetic retinal disease (DRD). With appropriate use, OCT-A characteristics and measurements have the potential to become new imaging biomarkers in managing and treating DRD. Major challenges include (a) provision of standardized outputs from different OCT-A instruments providing
\end{abstract}

standardized terminology to correctly interpret data; (b) the presence of artifacts; (c) the absence of standardized grading or interpretation method in the evaluation of DRD, similar to that already established in fundus photography; and (d) establishing how OCT-A might be able to provide surrogate markers to demonstrate blood retinal barrier breakdown and vascular leakage, commonly associated with DRD. In fact, OCT-A guidelines for DRD are still evolving. The outputs of quantitative OCT-A data offer a unique opportunity to develop tools based on artificial intelligence to assist the clinicians in diagnosing, monitoring, and managing patients with diabetes. In addition, OCT-A has the potential to become a useful tool for the evaluation of cardiovascular diseases and different neurological diseases including cogni- karger@karger.com www.karger.com/ore

Karger $\stackrel{\text { ' }}{5}$

GOPEN ACCESS
(C) 2021 The Author(s)

Published by S. Karger AG, Basel

This is an Open Access article licensed under the Creative Common Attribution-NonCommercial-4.0 International License (CC BY-NC) (http://www.karger.com/Services/OpenAccessLicense), applicable to the online version of the article only. Usage and distribution for commercial purposes requires written permission.
Correspondence to:

Stela Vujosevic, stela.vujosevic @ gmail.com 
tive impairment. This article written by the members of Diabetic Retinopathy expert committee of the European Vision Clinical Research network will review the available evidence on the use of OCT-A as an imaging biomarker in DRD and discuss the limits and the current application as well as future developments for its use in both clinical practice and research trials of DRD.

(C) 2021 The Author(s)

Published by S. Karger AG, Basel

\section{Introduction}

Imaging modalities play an important role in early diagnosis of diabetic retinopathy (DR) and diabetic macular edema (DME), in devising treatment protocols and evaluating the prognosis of visual outcome. Fundus color photography was developed to predict the progression of DR in the modified Airlie House Classification [1] using 2-dimensional markers in the Diabetic Retinopathy Study [2] and Early Treatment Diabetic Retinopathy Study. Simplified grading scales $[3,4]$ are more widely used in screening to detect DR at the appropriate stage in the disease process for treatment to be given. Scanning confocal ophthalmoscopes have been developed that capture a wider field and may be better in a nonmydriatic format, but the additional costs have so far limited their use in screening [5].

The opportunity for 3-dimensional image capture was first introduced on a large scale with the development of optical coherence tomography (OCT) that is widely used as an imaging biomarker for assessment and treatment of DME. The parameters most frequently used include central retinal thickness, central foveal subfield thickness, intraretinal cystoid spaces, hyperreflective retinal foci/ spots, disorganization of retinal inner layers, and subfoveal neurosensory detachment [6].

Fundus fluorescein angiography (FFA) was first described in 1960 and has been the main method used to examine the retinal circulation, despite the fact that it involves the intravenous injection of fluorescein dye, until the introduction of noninvasive OCT-angiography (OCT-A). OCT-A represents a revolution in the noninvasive evaluation of retinal and choroidal circulation (with a potential to become a new imaging biomarker in DR/DME), allowing visualization and study of retinal plexuses at different depths. When compared to FFA, OCT-A provides a more detailed view of the alterations of the foveal avascular zone (FAZ), disruption of the perifoveal capillary net, and better visualization of the microaneurysms in the deep capillary plexus (DCP) even if FFA is able to detect more microaneurysms $[7,8]$. However, OCT-A is unable to directly demonstrate the blood retinal barrier breakdown and vascular leakage, commonly associated with DR, but some approaches are under investigation in order to obtain this information noninvasively $[9,10]$. As with every new technique, OCT-A presents significant challenges in the correct understanding and interpretation of data, as well as the use of standardized terminology in order to uniform results and make them comparable across studies, thus increasing the translational relevance. The aim of this study was to review the available evidence on the use of OCT-A as an imaging biomarker in DR/DME and to better understand its potential and limitations, as well as future developments for its use in both clinical practice and research trials.

\section{OCT-Angiography Technique}

OCT-A is a novel, high-resolution, noninvasive, volumetric, imaging technique that allows for an easy and separate evaluation of retinal and choroidal circulation. OCT-A uses motion-contrast imaging to represent the erythrocyte movement in retinal blood vessels by comparing the decorrelation signal (differences in the backscattered OCT signal intensity or amplitude) between sequential OCT B-scans performed in the same area, in few seconds [11]. Although OCT-A cannot measure leakage, it can separately evaluate different retinal plexuses in the macula (superficial, intermediate, and deep capillary plexuses), the choriocapillaris (CC), choroid, and radial peripapillary capillary network [12-15].

Two types of OCT-A instruments are currently in use: spectral domain (SD-OCT-A) and swept source (SSOCT-A). The SD-OCT-A uses a broadband near-infrared super-luminescent diode as light source, a center wavelength of approximately $840 \mathrm{~nm}$, and a spectrometer as the detector. The SS-OCT-A uses a tunable swept laser as light source, a center wavelength of approximately $1,050 \mathrm{~nm}$, and a single photodiode detector. The higher scanning speed of SS-OCT-A allows for fewer motion artifacts, whereas the longer wavelength with greater light penetration into tissues allows for better visualization of choroid and CC $[16,17]$. Three ways of processing OCTA data are available: (1) based on the intensity of the OCT signal (e.g., OCT angiography ratio analysis, the splitspectrum amplitude-decorrelation angiography); (2) based on the phase of the OCT signal (e.g., Doppler or phase variance OCT); and (3) together as the complex 

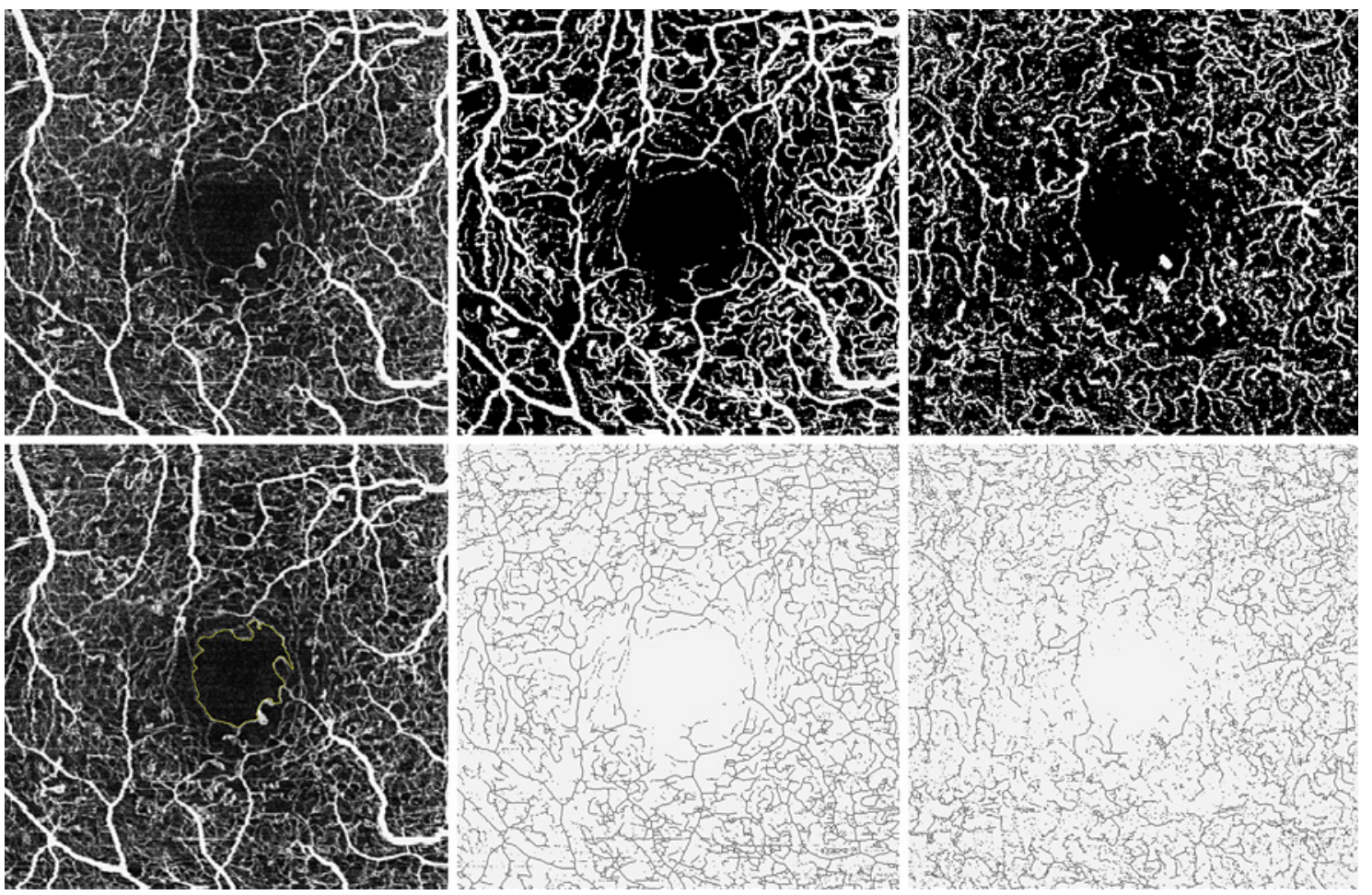

Fig. 1. Quantitative metrics evaluated on OCT-A images in the SCP and DCP in the eye with moderate nonproliferative diabetic retinopathy. The top left shows a $3 \mathrm{~mm} \times 3 \mathrm{~mm}$ slab of the SCP and the bottom left shows the FAZ delineation on the SCP that allows to calculate the following parameters: $\mathrm{FAZ}$ area $=0.301 \mathrm{~mm}^{2}$; FAZ perimeter $=2.952 \mathrm{~mm}$; and circularity index $=4 \pi \times$ area/perimeter $^{2}=0.4340539$; top middle and bottom middle show the binarized and skeletonized images of the SCP using the Otsu method; top right and bottom right show the binarized and skeletonized images of the DCP of the same eye. Binarized images are used for determination of perfusion density, and skeletonized images are used for determination of vessel density. OCT-A, optical coherence tomography angiography; SCP, superficial capillary plexus; DCP, deep capillary plexus; FAZ, foveal avascular zone.
OCT signal (e.g., OCT microangiography-complex [OMAG C], which incorporates differences in both the phase and intensity information contained within sequential B-scans) [17].

\section{Qualitative and Quantitative Metrics in OCT-A}

OCT-A allows for both qualitative and quantitative analyses of the retina and the choroidal vasculature. The qualitative analysis aims to evaluate the shape of the FAZ; the presence of retinal lesions (such as nonperfusion areas, microaneurysms, new vessels, collaterals, cystoid space, and morphology of retinal capillaries); and the presence of choroidal lesions (detection of choroidal neovascularization, its type and morphology (medusa, sea fan, glomerular, and dead tree), and CC structure analysis). It is highly dependent on accurate segmentation and the absence of projection artifacts $[15,18-21]$.

The quantitative analyses require the application of the threshold in order to obtain the binarized images. The quantitative metrics include the evaluation of the area, perimetry, and circularity index (CI) of the FAZ; and the retinal perfusion parameters (perfusion and vessel density, vessel diameter index, perfused capillary density, and vascular complexity parameters) [12, 22-25] (Fig. 1).

Nomenclature of Quantitative Parameters

FAZ quantitative parameters include the area, the perimeter (expressed in $\mathrm{mm}$ ), and the CI (defined by the equation $\left[4 \pi \times\right.$ area] $/$ perimeter $^{2}$ ). The $\mathrm{CI}$ is the expression of the regularity of a shape: the more its value is closer to 1 , the more the shape is similar to a perfect circle [26].

Perfusion density (PD) is a unit-less measure determined on binarized images and defined as the total area of perfused vasculature (pixels over the selected threshold) per unit area in a region of interest. Vessel density (VD), expressed in $\mathrm{mm}^{-1}$ on skeletonized images, is defined as the total length of perfused vasculature per unit 
area in a region of interest. Of note is the fact that the term "VD" is frequently used to describe PD.

Vessel diameter index is defined as the average vessel caliber (total vessel area on binarized images/total vessel length on skeletonized image) [27]. Perfused capillary density is defined as the percentage of the capillary area divided by the total analyzed area, once noncapillary blood vessel areas have been subtracted, on full vascular slab [22]. Fractal dimension (FD) is a mathematical parameter that describes the degree of complexity of a biological structure, using the box-counting method on the binarized image [23]. Number of branches and total branch length (total sum of the single branches' length in the analyzed area) on the skeletonized image were evaluated in both the peripapillary and macular area $[12,25]$. In order to evaluate CC perfusion, the term "signal voids" is used, defined as the dark areas where CC flow is undetectable due to either areas of real CC nonperfusion (flow voids) or areas where flow signal strength is below the decorrelation threshold $[28,29]$.

\section{The Importance of Standardization of Parameters in \\ OCT-A Image Evaluation}

While OCT-A has brought great advancement in our understanding of physiology and clinical findings, a consensus on terminology and standardized nomenclature is still missing. For example, decreased or absent flow due to DR has been termed as "flow void" [30], "capillary nonperfusion" [31], or "impaired capillary perfusion" [32]. The terms "PD" and "VD" have been used confusingly to quantify the percentage area occupied by perfused binarized vessels [33]. Moreover, the method of thresholding and adjustments in contrast and brightness, as well as the use of high-resolution versus high-speed mode, can significantly impact quantitative data analyses [34-37]. This makes direct comparisons among different studies and populations impossible. For the longitudinal analyses, the same parameters should be used including the instrument, angiocube size, pattern, and scan location as well as the algorithm [36].

OCT-A manufacturers use different units of measurement, different segmentation strategies, and variable FAZ assessment, making a reliable comparison of OCT-A data between different instruments impossible [38]. In clinical trials, quantitative data obtained with different instruments should not be amalgamated. For example, only the FAZ area seems to be a useful parameter for OCT-A image analysis when using different machines, whereas VD and FD show a significant variability across devices [39]. Consensus terminology is under development and will

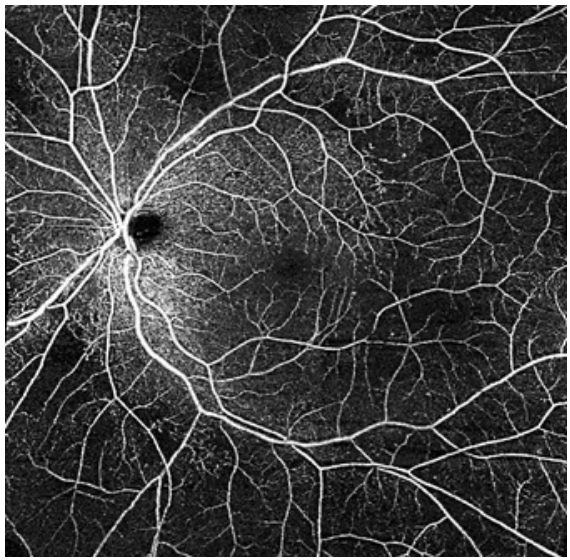

Fig. 2. OCT-A image of the left eye of the patient with moderate nonproliferative diabetic retinopathy (montage of 5 scans, each $8 \times 8 \mathrm{~mm}$, covering an area of approximately $14 \mathrm{~mm} \times 14 \mathrm{~mm}$ ) obtained with Zeiss Cirrus HD-OCT 5000 Angioplex (Zeiss Meditec, Dublin, CA, USA), showing areas of capillary rarefaction temporal to the macula, and outside of the arcades. Details of the FAZ and the perifoveal area are better visualized with smaller scans that have higher resolution. OCT-A, optical coherence tomography Angiography; FAZ, foveal avascular zone.

allow to simplify clinical and scientific work by clarification and standardization, as well as to improve the measurement accuracy [38].

\section{The Role of Imaging Biomarkers in DR}

Biomarkers, as variables of clinical relevance that can be objectively measured and monitored, are critical tools to identify individuals at risk of developing a disease, those in whom the disease will progress and those with a specific response to a therapeutical intervention [40]. In the context of DR, distinct markers have been proposed as biomarkers for the pathological events, such as circulating biomarkers (obtained from serum sampling), local biomarkers (obtained from eye-related biologic fluids), and imaging biomarkers (reviewed in Refs. [41, 42]). Biomarkers are also seen as the key to personalized medicine, treatments individually tailored to specific patients for highly efficient intervention in disease processes.

It is now clear that systemic markers of diabetes such as disease duration, poor glycemic control, increased blood pressure, and lipid levels are relevant risk factors, but they do not necessarily identify DR worsening accurately enough $[43,44]$. These observations led to the identification of different phenotypes of progression [45] based on the characteristics of the retinal lesions. It is, 


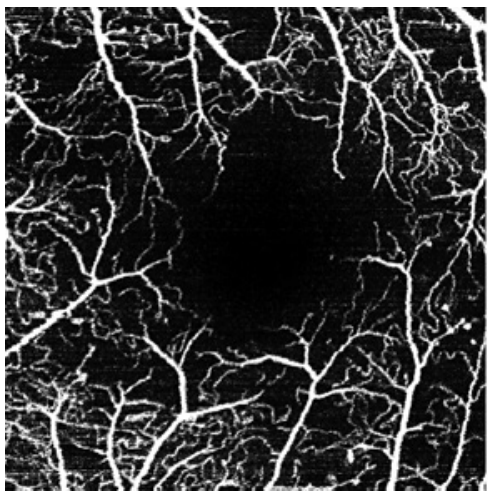

Fig. 3. OCT-A images of the macula ( $3 \mathrm{~mm} \times 3 \mathrm{~mm}$ slab) of the same eye as in Fig. 1, obtained with the Spectralis OCT-A instrument (Heidelberg Engineering, Heidelberg, Germany), showing details of the FAZ and the perifoveal capillary network. The left image shows the SCP; the middle image shows the ICP; and the

therefore, fundamental to be able to identify the retinal lesions and their dynamics in the earlier stages of DR and correlate their occurrence to the progression of any stage of DR to vision-threatening complications $[46,47]$.

A recent 5-year prospective observational longitudinal study reported that ocular risk markers are more informative than systemic risk markers for predicting, in patients with well-controlled diabetes and mild DR, the eyes at risk for developing vision-threatening complications, namely, center-involved macular edema (CIME), clinically significant macular edema (CSME, defined as by the Early Treatment Diabetic Retinopathy Study group on clinical examination), or proliferative diabetic retinopathy (PDR) [48]. Development of DME, either CSME or CIME, and PDR was associated with baseline microaneurysm turnover (MAT), CRT, and GCL + IPL thickness and VD metrics on OCT-A [48].

The use of noninvasive imaging approaches has gained much relevance in the identification of possible DR biomarkers. The rate of MAT, assessed in clinical practice in a field 2 color fundus photograph, using the automated Retmarker screening software (Retmarker S.A.), has been widely shown to be associated with the development of DME [14-16]. This method has received a letter of support from the European Medicines Agency as the first biomarker of DR progression [49].

The use of multimodal imaging approaches, and particularly OCT and OCT-A, facilitates the identification of different pathways of DR and their relative relevance in individual patients [50]. OCT-A has gained a critical position in the evaluation of DR [51] as it allows the identification and quantification of retinal capillary closure, the right image shows the DCP. There is an irregular and enlarged FAZ in all the plexuses as the rarefaction of the perifoveal capillary network. OCT-A, optical coherence tomography angiography; SCP, superficial capillary plexus; DCP, deep capillary plexus; FAZ, foveal avascular zone; ICP, intermediate capillary plexus.

feature that better correlates with the clinical signs of nonproliferative DR (NPDR) in its main stages and progression $[50,52]$.

\section{OCT-A as a Diagnostic Biomarker of DR}

A diagnostic biomarker detects or confirms the presence of a disease or a condition of interest or identifies an individual with a subtype of the disease [53]. Many of the common features of DR, as seen on FFA, including microaneurysms, neovascularization, capillary closure, and regions of nonperfusion have been extensively studied and described using OCT-A (Figs. 2, 3). OCT-A is, therefore, retriable and a more sensitive procedure for detailed morphological evaluation of central macular vascular changes in diabetes [8]. There is also evidence from different studies that OCT-A measurements may reveal the presence of DR before it is clinically detectable $[54,55]$.

VD provides a quantitative metric of capillary closure or dropout that has shown correlation with severity of DR and its progression, being strongly associated with the clinical features of nonproliferative DR [56, 57]. Durbin et al. [58] showed that VD measured in the superficial capillary plexus (SCP) can discriminate healthy from DR eyes. A reduction in retinal VD in both the SCP and DCP, mainly at the macular area, has been correlated to visual acuity (VA) and may be a potential indicator for macular ischemia and vision loss during disease progression. The regional distribution of retinal capillary changes may be particularly relevant in staging DR $[19,59]$. Studies with wide-field FFA have also shown that peripheral nonperfusion lesions are associated with higher risks of DR progression [60]. 
More recently, SS-OCT-A instruments enabled visualization of retinal vasculature over larger fields of view, like $12 \times 12 \mathrm{~mm}$ or $15 \times 9 \mathrm{~mm}$, instead of the common $3 \times 3 \mathrm{~mm}$ perifoveal acquisitions. Combining the results of $3 \times 3 \mathrm{~mm}$ and $15 \times 9 \mathrm{~mm}$ acquisitions, Santos et al. [61] distinguished patients from 2 major stages of NPDR: mild retinopathy (ETDRS 20-35), with capillary closure limited to the perifovea; and moderate-to-severe NPDR (ETDRS 43-53) with a significant increase of retinal capillary closure in more peripheral regions of the retina. Similar findings were also reported by Tan et al. [62]. In summary, OCT-A allows quantitative measurements of the vascular changes occurring in DR, with particular relevance for measurements of VD or PD indicative of capillary closure, suggesting a promising role of OCT-A as a diagnostic biomarker of DR [63].

\section{OCT-A as a Diagnostic Biomarker in Diabetic Macular Edema}

As recent results confirm that different disease processes may be involved in the development of CSME and CIME, it is crucial that these outcomes should not be analyzed together. CIME and CSME are associated with different retinopathy phenotypes, with CSME occurring mainly in eyes with decreased VD and abnormal MAT, indicating the presence of ischemic changes [64]. Retinal thickness, indicating the presence of edema, is the only risk marker that is present in both CIME and CSME, confirming previous observations that it may be a predictor for development of DME [65].

However, in DME, the use of OCT-A presents more limitations due to the high rates of segmentation errors caused by changes in retinal layers' architecture and artifacts on the vascular network caused by the presence of intraretinal cystoid spaces [19]. Improved accuracy of automated segmentation is required and would be very beneficial for DME evaluation in the future. However, OCT-A is already useful for characterizing the pathogenesis of DME and identifying the presence of diabetic macular ischemia, an important cause of vision loss, and an indicator of poor response to DME treatment [14]. Narrowing and occlusion of retinal capillaries around the foveal area, especially in the DCP, are the most important signs of diabetic macular ischemia, showing a strong correlation with photoreceptors disruption and consequently with VA decrease [66].

\section{OCT-A as a Prognostic Biomarker of DR Disease}

Progression

By definition, a prognostic biomarker is used to identify the likelihood of a clinical event, disease recurrence, or disease progression in patients with a disease or medical condition of interest [67]. In the case of DR, its progression is defined by the 7 field ETDRS grading, which has been well validated [68].

OCT-A allows the detection of microaneurysms and nonperfusion in patients with DR, a helpful addition in assessing disease progression, as proposed by Couturier et al. [7]. Similarly, Hwang et al. [69] determined that OCT-A can clearly delineate capillary dropout and retinal new vessels, highlighting a possible role in monitoring for ischemic and proliferative changes among eyes with DR.

In a 5-year longitudinal study, it was shown that ocular biomarkers such as MAT and capillary closure are biomarkers of DR progression [54]. Pairwise comparisons indicated that a higher MAT was present in eyes with 2-or-more-step worsening of DR versus any other category. Importantly, capillary closure, identified by a decrease in VD determined at a 5-year follow-up, decreased in all ETDRS levels. This decrease showed a significant correlation with DR severity progression [54].

Recently, data from a 3-year longitudinal study showed that the retinal capillary closure increases with DR progression, in contrast with edema and neurodegeneration, which remained stable throughout the whole follow-up. When looking for factors associated with capillary closure on a univariate regression analysis, MAT and GCL + IPL thinning (inner ring layer) were found to be significantly associated with VD. However, in a multivariate regression analysis (including also demographic and systemic features), only MA turnover showed a statistically significant association with VD decrease (capillary closure). Furthermore, during this period, 1- or 2-step worsening of DR was associated with the presence of capillary closure, suggesting that only increased capillary dropout is an indicator of DR severity progression (RETINA submitted 2021, Marques et al. [54]). OCT-A metrics of retinal capillary closure obtained in a noninvasive manner with repeated examinations appear to be more informative than systemic risk markers for predicting, in eyes of patients with well-controlled diabetes and mild retinopathy, which ones are at risk for developing vision-threatening complications, being a promising candidate as a biomarker of DR severity progression, and are expected to impact management of DR.

\section{OCT-A Role as an Alternative to ETDRS Grading}

The ETDRS classification is based on the presence of different features identified by fundus photography. These alterations are considered to be related to the presence of capillary closure and ischemia [70]. It is, there- 
fore, reasonable to think that capillary closure, if reliably quantified and measured, may be an appropriate indicator of changes identified by fundus photography and become an alternative to the ETDRS classification.

Considering that only 2-step changes in ETDRS severity grading have been shown to be clinically significant, it was proposed that ETDRS grades 10 and 35 correspond to no and mild DR and ETDRS grades 43-53 to moderate and severe DR. Grades 43-53 may represent objectively and more accurately the real progression of NPDR [61]. The use of SS-OCT-A, with combined protocols, capable of detecting early capillary closure in the perifoveal region and, later, on the disease process capillary closure in the midperiphery, may offer a practical and simple to use alternative to the laborious and demanding ETDRS grading process, which classifies a complex set of parameters. The results here reviewed may give, indeed, a practical basis for the International Classification of Diabetic Retinopathy [3].

\section{OCT-A as a Predictive Biomarker of DR/DME}

\section{Treatment Response}

Predictive biomarkers discriminate those who will respond or not respond to treatment and are therefore important in the design and conduct of clinical trials [67]. OCT-A may be useful to identify some predictive biomarkers of DR and DME treatment response, especially if integrated with information obtained with OCT. Several studies reported the changes in OCT-A parameters before and after treatment, focusing on the possible vascular reperfusion after the intravitreal injections of antivascular endothelial growth factor (VEGF), steroids, and subthreshold micropulse laser, but very few of them reported specific OCT-A biomarkers to predict the treatment response comparing different drugs [6]. Currently, OCT-A parameters considered as biomarkers of DME treatment response are the characteristics of the FAZ in different retinal layers in terms of size and circularity index, the characterization of MAs, their visibility and internal reflectivity, number and location, the measurement of VD, NPAs, and vessel tortuosity.

\section{Anti-VEGF Drugs}

An impairment of the DCP, characterized by the presence of a great number of MAs, a lower VD, and FAZ enlargement, is reported as a biomarker of poor response to anti-VEGF treatment [71-73]. Even the internal reflectivity of MAs through OCT and OCT-A evaluation correlates with macular ischemia, suggesting that the hyporeflective MAs located in the DCP could be biomarkers of poor response to anti-VEGF [74].

OCT-A as Imaging Biomarker in DRD
Another OCT/OCT-A parameter that influences the VA improvement after treatment is the presence of giant intraretinal cysts $(>200 \mu \mathrm{m})$ on OCT; these cysts on OCT$A$ are often characterized by a hyperreflective internal signal named suspended scattering particles in motion; the giant intraretinal cysts and the suspended scattering particles in motion correlate with macular ischemia and could be an indirect expression of poor visual prognosis [75].

More recently, a topographical OCT-A analysis considering different macular regions showed that the parafoveal VD in the SCP at baseline could be an independent predictor for VA improvement after the loading dose with ranibizumab, in the multiple regression model with adjustment for central macular thickness and ellipsoid zone disruption [73]. In the same study, a higher VD at the level of SCP seemed to determine a greater VA improvement after treatment, but further studies are needed to validate the role of $\mathrm{VD}$ as a biomarker of response to ranibizumab injections [73]. Another recent study reported a better VA after aflibercept intravitreal injections for DME in eyes with a larger baseline retinal vascular area (defined as the percentage of the entire area occupied by large vessels and microvasculature) in SCP and DCP [76]. Furthermore, the resolution of MAs may be associated with better VA and resolution of DME after intravitreal aflibercept [76].

Another important OCT-A parameter to predict the treatment response is the FAZ analysis. It was reported that a larger baseline FAZ area correlates with poor response to anti-VEGF treatment $[71,72]$.

Regarding the possible role of anti-VEGF drugs on the retinal reperfusion, conflicting results were reported. The VD is the most used OCT-A parameter for quantification of retinal blood flow [7].

In a previous study by Sorour et al. [77], no correlations between anatomic response and VD measurement were found after a single dose of anti-VEGF, suggesting that the anti-VEGF effect on macular perfusion may not be a direct change in microvascular flow. Also, OCT-A data from Couturier et al. [78] showed no changes in NPAs and no reperfusion of small retinal vessels in NP areas on SS-WF OCT-A and UWF-FFA after anti-VEGF therapy, although the DRSS improved and neovascularization regressed. Despite theis evidence, a recent study [79] showed that an intensive aflibercept treatment may be effective in reducing NPAs in patients with DR without DME, reporting that an important vascular leakage was significantly associated with retinal reperfusion after aflibercept treatment. 


\section{Steroids}

As for the anti-VEGF, also for steroids, it is important to integrate OCT and OCT-A data as imaging biomarkers of treatment response. The FAZ area alone and combined with ellipsoid zone disruption, adjusted for age, could be considered a predictor of VA in macular vascular disease as DR and DME, in an OCT/OCT-A model [80].

Recently, Vujosevic et al. [6], compared the effects of ranibizumab versus dexamethasone on several OCT and OCT-A inflammation biomarkers reporting a greater reduction of hyperreflective foci in the inner retina, central macular thickness, DRIL extension, cysts area in DCP, FAZ CI, and VD DCP in the dexamethasone group; this evidence suggests that a detailed analysis of inflammation signs at baseline could guide the treatment choice, potentially useful for final prognosis. By the analysis of OCT-A, no changes were described in NPAs and in the VD at the level of DCP at 2- and 4-month follow-ups after dexamethasone [81], but an early decrease in NPAs was reported, suggesting a possible precocious drug-related reperfusion.

Can OCT-A Be Useful for Evaluation of the Preclinical Stage of DR and Its Progression in Type 1 and Type 2 Diabetes Mellitus?

It has been reported that specific neural and microvascular abnormalities might characterize types 1 and 2 diabetes mellitus (DM) (T1DM/T2DM), even before clinical signs of DR are visible on OCT and OCT-A. The inner retinal layer (ganglion cell layer) was reported to be significantly thicker in T1DM versus T2DM in the central 1 $\mathrm{mm}$, after adjusting for age and DM duration [15]. In those with T1DM versus controls, the area of FAZ was significantly larger in SCP and DCP; such a difference in FAZ was only found in DCP for those between T1DM versus T2DM only in DCP. The number of focally dilated endings of the capillaries was higher in T1DM versus controls in both SCP and DCP; and in T2DM versus controls only in DCP. Perifoveal capillary loss in SCP had the highest correlation to the inner retinal layer thickness in both DM types [15]. It has been reported that in T1DM, DCP impairment may be a precursor of retinal vascular damage, even before any clinical signs of DR, suggesting that the perifoveal capillary dropout represents an early damage [82]. Based on current evidence, OCT-A biomarkers associated with DR progression are specific to the type of diabetes, and so far only DCP impairment might be considered as a common biomarker for progression. Sun et al. [83] explored the role of impairment at the level of DCP in T2DM, expressed as FAZ area, FD, and
$\mathrm{VD}$, as a factor associated to the progression of $\mathrm{DR}$ in 2 years. On the contrary, VD at SCP seems to be strictly associated to the development of DME during the followup [78]. Recently, Scarinci et al. [84] reported that in T1DM, a decrease in VD of DCP and more specifically of the ICP during the early stages of the disease is the most robust parameter in providing objective imaging biomarkers to monitor the clinical progression of NPDR in 2 years' follow-up (Table 1 ).

\section{OCT-A and Systemic Status: The Endocrinologist's}

Point of View

The influence in vascular changes determined by OCT-A related to systemic conditions such as glycemic control, cardiovascular risk factors, and other comorbidities is yet to be highlighted. Acute hyperglycemia has been shown to impact neurovascular coupling in human eyes [85], and it has been recently reported that it significantly decreases the DCP vessel density in the dark [86]. Therefore, in pathological states, such as diabetic retinal disease (DRD), where the vasculature is already attenuated, photoreceptors might be more susceptible to ischemia. This finding opens up a new research field addressing the need to examine the impact of glycemic variability on OCT-A. In addition, the effect of the rapid reduction of blood glucose levels on retinal microvasculature and the potential differences depending on the antidiabetic drug used are questions that merit further investigation.

Little is known about the influence of cardiovascular risk factors on OCT-A findings. However, it seems that hypertension reduces $\mathrm{VD}$ and $\mathrm{PD}$ and increases FAZ, thus aggravating the effects of chronic hyperglycemia [87, 88]. To the best of our knowledge, there are no reports about the impact of lipids or smoking habit on OCT-A.

Regarding comorbidities, a recent study provided evidence that patients with acute coronary syndrome showed a lower inner vessel length density in comparison with those patients without cardiovascular disease [89]. This finding supports the link between micro- and macroangiopathy in the setting of diabetes complications. The influence of other comorbidities such as obesity has not been examined. However, it should be noted that sleepapnea syndrome is frequent in patients with T2DM with obesity and is associated with the hypoxia/hypercapnia and the transient increase of blood pressure, and these factors can all affect retinal microcirculation.

Finally, a general remark should be made. It is frequently forgotten that the diagnosis of diabetes is based on an underlying assumption that there is a clear glycemic threshold that separates persons at high and low risk of DR 
Table 1. Summary of OCT-A biomarkers in diabetic retinal disease

\begin{tabular}{ll}
\hline OCT-A biomarker & Definition \\
\hline Vessel metrics & VD: total length of perfused \\
& vasculature per unit area in a \\
& region of interest; quantitative \\
& metric of capillary closure or \\
& dropout \\
& PD: total area of perfused \\
& vasculature (pixels over the \\
& selected threshold) per unit \\
& area in a region of interest
\end{tabular}

Biomarker
classificatio

Correlation to diabetic retinal disease Corr eyes with

Extent of capillary closures distinguishes mild NPDR (limited to the perifovea) from moderate to severe NPDR (significant increase of retinal capillary closure in more peripheral retina) CSME occurs mainly in eyes with decreased VD and abnormal MAT, indicating the presence of ischemic changes Narrowing and occlusion of retinal capillaries around the foveal area, especially in the DCP, indicate diabetic macular ischemia A significant increase in the area of capillary loss and the presence of tortuosity/beadings in the perifoveal area in both SCP and DCP in DM type 1 and type 2 without clinical signs of DR versus controls; and higher number of focally dilated endings of the capillaries in DM type 1 versus controls in both SCP and DCP; and in DM type 2 in DCP

Prognostic VD is correlated with severity of DR and its progression, and strongly associated with the clinical features of nonproliferative DR

OCT-A can clearly delineate capillary dropout and retinal new vessels, highlighting a possible role in monitoring for ischemic and proliferative changes among eyes with DR Decrease in VD from baseline is correlated with DR severity progression

Predictive Lower VD in the DCP corresponds to poor response to antiVEGF treatment

Parafoveal VD in the SCP at baseline could be a predictor of visual improvement after loading dose with ranibizumab Higher VD at the SCP may be correlated with greater visual improvement after treatment with ranibizumab Better VA is reported after aflibercept intravitreal injections for DME in eyes with a larger baseline retinal vascular area in SCP and DCP

\begin{tabular}{lll}
\hline FAZ size & $\begin{array}{l}\text { FAZ size expressed in square } \\
\text { millimeters (area) or in } \\
\text { millimeters (perimeter) }\end{array}$ & $\begin{array}{l}\text { Diagnostic } \\
\text { Predictive }\end{array}$ \\
&
\end{tabular}

FAZ enlargement in both SCP and DCP in DM type 1

without clinical signs of DR versus controls

FAZ enlargement in the DCP corresponds to poor response to anti-VEGF treatment

Larger baseline FAZ area correlates with poor response to anti-VEGF treatment

FAZ area alone and combined with EZ disruption, adjusted for age, could predict VA after steroid treatment in DR and DME

\begin{tabular}{llll}
\hline FAZ CI & $\begin{array}{l}\text { Regularity of FAZ shape (the } \\
\text { more its value is closer to 1, } \\
\text { the more the shape is regular } \\
\text { and similar to a perfect circle) }\end{array}$ & Predictive & $\begin{array}{l}\text { There is a greater reduction in FAZ circularity index in eyes } \\
\text { receiving dexamethasone treatment compared with anti- } \\
\text { VEGF }\end{array}$ \\
\hline $\begin{array}{l}\text { Microaneurysm } \\
\text { internal reflectivity }\end{array}$ & Predictive & $\begin{array}{l}\text { Hyporeflective MAs located in the DCP may be associated } \\
\text { with poor response to anti-VEGF treatment }\end{array}$ \\
\hline $\begin{array}{l}\text { Giant intraretinal } \\
\text { cysts }\end{array}$ & $\begin{array}{l}\text { Cystic changes }>200 \mu \mathrm{m} \text { in size } \\
\text { and often characterized by a } \\
\text { hyperreflective internal signal } \\
\text { named SSPiM (suspended } \\
\text { scattered particles in motion) }\end{array}$ & Predictive & $\begin{array}{l}\text { Presence of giant intraretinal cysts and SSPiM correlates with } \\
\text { macular ischemia and could be an indirect expression of poor } \\
\text { visual prognosis after treatment with anti-VEGF }\end{array}$ \\
\hline
\end{tabular}

SCP, superficial capillary plexus; DCP, deep capillary plexus; FAZ, foveal avascular zone; CSME, clinically significant macular edema; MA, microaneurysm; MAT, microaneurysm turnover; VA, visual acuity; EZ, ellipsoid zone; VD, vessel density; PD, perfusion density; SSPiM, suspended scattering particles in motion; VEGF, vascular endothelial growth factor; CI, circularity index; DME, diabetic macular edema; DR, diabetic retinopathy; DM, diabetes mellitus; NPDR, nonproliferative DR.
Studies

Durbin et al. [58]

Santos et al. [61]

Marques et al. [64]

Scarinci et al. [66]

Vujosevic et al. [15]

Rodrigues et al. [56]

Samara et al. [57]

Hwang et al. [69]

Marques et al. [54]

Parravano et al. [74]

Lee et al. [72]

Hsieh et al. [73]

Busch et al. [76]

Vujosevic et al. [15]

Parravano et al. [74]

Lee et al. [72]

Hsieh et al. [73]

et al. [80]

DOI: $10.1159 / 000518620$ 
[90]. Current OCT-A evidence suggests that the arbitrary cutoff of $7 \mathrm{mmol} / \mathrm{L}$ for blood glucose in diagnosing diabetes might not identify all of those at risk of vascular complications; changing the diagnostic criteria will open a new era of management of diabetes and its complications.

\section{OCT-A Image Evaluation: Clinical and Reading Center Perspective}

One of the major limitations of OCT-A currently lies in its inability to evaluate leakage from retinal vessels. In addition, the presence of artifacts significantly impacts the evaluation of both the retina and the CC in many cases. Moreover, the blood flow is detected at a certain speed, but measurements of blood flow velocity or flow volume are still unavailable and consequently those vascular structures with very slow blood flow may not be identified at all. Technical improvements are crucial to increase the accuracy of lesion identification such as microaneurysms, new vessels, and small vessels adjacent to intraretinal cysts. Vascular reconstruction with 3D OCT-A vessel visualization may also be helpful to clinicians for detecting microvascular changes [91].

\section{The Pitfalls in OCT-A Image Evaluation}

When interpreting OCT-A images, physicians need to understand and be aware of the potential source and meaning of the artifacts, not caused by blood flow. Possible causes include the following [92]: (1) The image acquisition process is longer than for structural OCT, as the same area needs to be scanned multiple times at a high A-scan rate. (2) Intrinsic ocular properties or pathologies such as media opacities of the lens or vitreous may obscure visualization of the retina, while subretinal hemorrhage may not allow visualization of the underlying choroid. Signal attenuation caused by opacities can impede the ability to display and identify flow. (3) Eye movements can cause significant changes from one B-scan to the other and hereby falsely appear as vessel flow in successive images. (4) Data processing is another possible source of artifacts: low signal or random noise can cause "flow" artifacts; hence, strong OCT signals and masking or thresholding methods can improve reliability. En face images allow for visualization and evaluation of vascular plexuses and rely on layer segmentation, which is frequently incorrect in cases of altered retinal anatomy, such as in DME. The OCT-A vascular images are then incorrect. Hence, the interpreting physician should ensure correct segmentation(Fig. 4).
Challenges in DME Evaluation and How to Overcome Them

Stable fixation is needed in order to ensure high-resolution image acquisition. Reduced VA due to DME can impair a patient's ability to maintain stable fixation and hereby reduce image quality and produce motion artifacts [93]. Using a higher scan speed at the expense of image resolution can improve the acquisition process. Edematous thickening (retinal cysts) as well as ischemic retinal thinning can both impact slab segmentation accuracy and cause erroneous en face images [94]. Manual review and corrections may be needed to ensure proper segmentation. Advanced algorithms may improve performance and reduce these artifacts $[6,95,96]$.

\section{Choriocapillaris Evaluation in $D R$}

Significant choroidal changes have been associated with diabetes even in the early stages of DR, but the evaluation of this highly vascularized ocular structure using different imaging modalities, such as indocyanine green angiography, ultrasonography, and more recently with enhanced depth imaging OCT and SS-OCT, is limited. OCT-A allows for the first time a selective in vivo CC visualization in humans [97]. SD-OCT-A and SS-OCT-A both provide CC visualization, even if SS-OCT-A allows for better visualization as described in paragraph 2. Different software algorithms are in use for CC visualization and quantitative analysis (OMAG, split-spectrum amplitude-decorrelation angiography, and OCT-A ratio analysis) [30], shown to provide comparable results [98]. One of the major limitations of CC visualization remains that OCT-A images have a limited capability to display the intervascular spaces. Multiple en face OCT-A image averaging offers a solution by transforming the images from a granular appearance to a level where the intervascular spaces can be resolved [99, 100]. Therefore, more accurate methodology is needed to evaluate and quantify choroidal vasculature, and future improvements of SS-OCT-A may represent the correct approach [7]. Recent articles reported the presence of choroidal changes using this technology in patients with diabetes with different stages of disease, namely the presence of choroidal flow deficits [101, 102].

\section{The Reading Center Perspective: Manual Versus}

Automatic Evaluation and Agreement on Protocols in Clinical Trials

As the manual interpretation of OCT-A is subjective and prone to interobserver variability, commercial OCTA suppliers have developed proprietary software capable of quantitatively measuring vessel metrics to provide a 
Fig. 4. OCT-A images of the macula and corresponding OCT B-scan showing different types of artifacts. Top row: segmentation artifacts; second raw: motion artifacts; third raw: projection artifacts; bottom raw: low signal due to media opacities (cataract). OCT-A, optical coherence tomography angiography.
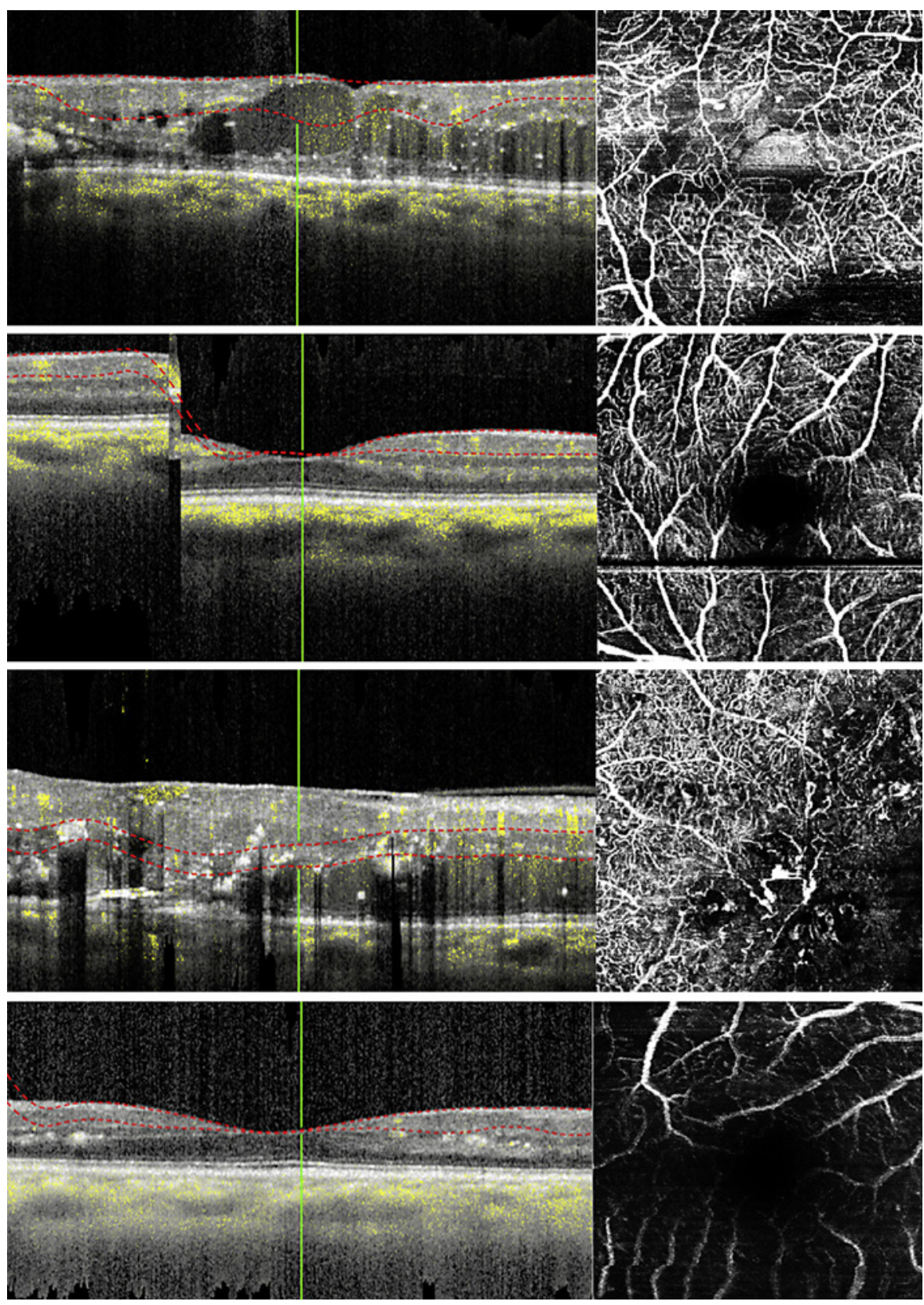

more objective method of evaluating OCT-A images. These metrics include VD, PD, and FAZ area. OCT-A metrics of the DCP can predict the progression of DR, while vessel metrics of the SCP can predict the development of DME [83]. This quantitative analysis software, however, is yet to be harmonized among the various commercially available OCT-A technologies, and therefore, there is currently no consistent automated OCT-A image grading. Each proprietary software uses its own algorithm; therefore, significant variations exist between the commercially available OCT-A devices [103]. How much these differences might contribute to clinical decisionmaking processes is not known at present. Table 2 summarizes the specifications and parameters measured by the various commercially available OCT-A devices.

Despite the recent developments in OCT-A technology and studies supporting its use in evaluation of DRD, the currently available literature provides no standardized grading or interpretation method for OCT-A in such eyes. Unlike fundus photography where established in- 


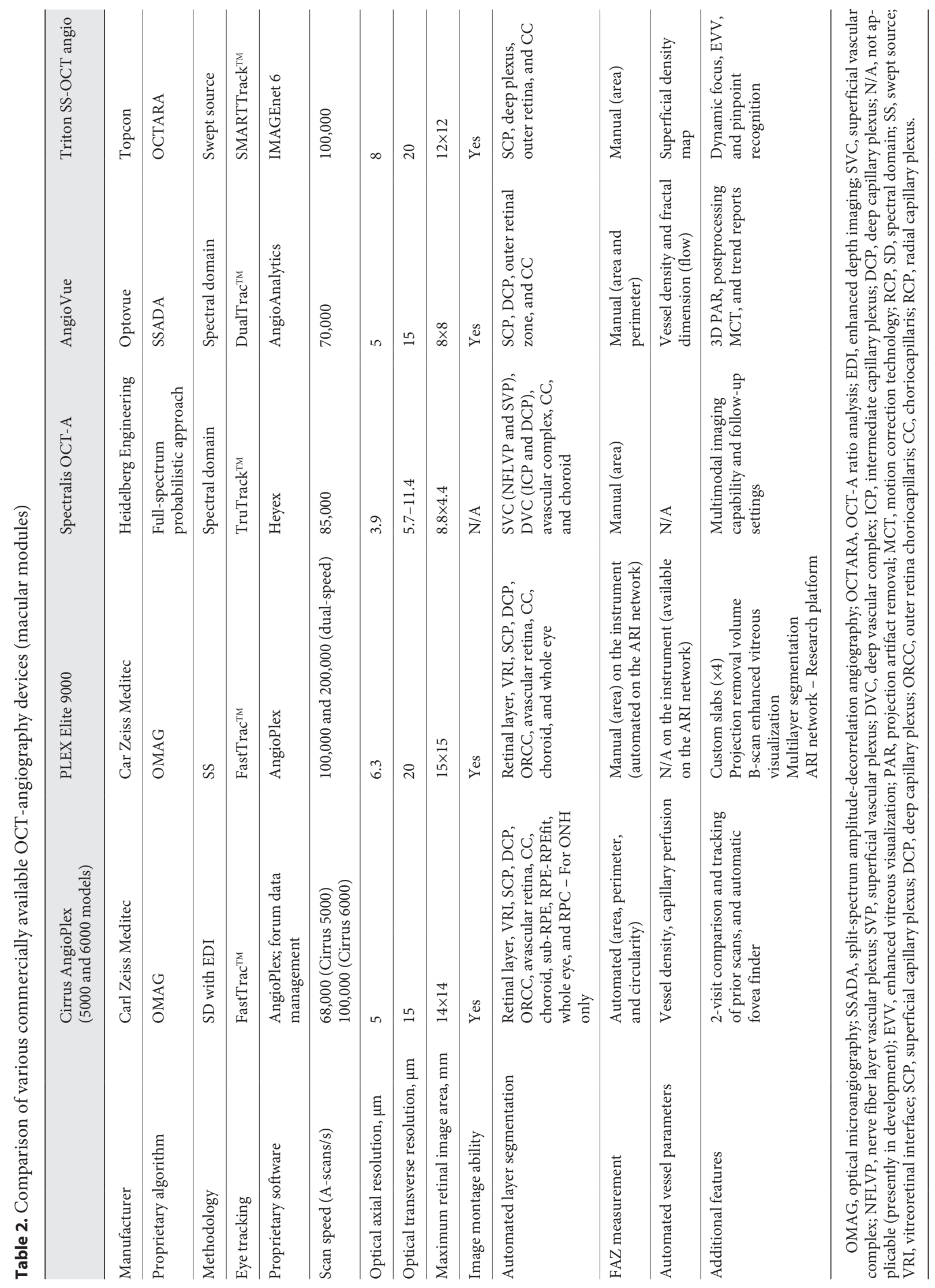


ternationally accepted grading guidelines exist, OCT-A guidelines for DR are still evolving. Furthermore, a study by Parrulli et al. [104] showed a high variability in the sensitivity, specificity, and positive predictive value among the different OCT-A devices in evaluating microaneurysms secondary to DR. Diabetic eye disease affects both the macula and the periphery, and so the limited field of view in OCT-A of the current devices limits its ability to examine the vascular changes in the periphery [103]. Wide-field OCT-A showed some promising results; however, limited availability means that its impact on large-scale diabetic eye screening and treatment programs is still unknown.

In order for OCT-A to be successfully integrated into current DR imaging programs, it is crucial that standardized protocols in image acquisition and interpretation be employed. This will provide image reproducibility, will make validation of diagnosis and treatment outcomes possible, and will make monitoring of changes over time more reliable and uniform. Image quality control mechanisms are also necessary for the effective use of OCT-A in DR evaluation and multicenter clinical trials [105]. Greig, Duker, and Waheed [106] offered a guide for a more effective OCT-A image analysis by assessing the (1) en face OCT-A image, (2) corresponding B-scans with vessel flow overlay, and (3) structural en face image for each retinal slab using a step-by-step method. As consensus on the elements of quality assurance and interpretation is still lacking, the utility of OCT-A for clinical trials and subsequent centralized reading center image analysis are also limited at present.

Despite limitations, OCT-A provides an additional and more detailed information than other imaging modalities on the retinal microvasculature in DR, although only on a small, but functionally vital area of the eye. To fully transition from being a research tool into a clinical aid for DR screening and treatment evaluation, OCT-A needs to further improve on certain aspects including the area of scan, standardization of algorithms, image acquisition and quality control, and appropriate training for clinical interpretation. Further discussions on the applicability of OCT-A in DRD are likely to continue in the near future.

\section{Future Perspective in OCT-A: Evaluation of Nonocular Disease in DM}

Most articles published on OCT-A in DR failed to analyze the so-called fourth retinal capillary layer - the radial peripapillary capillary plexus - which may be ob- served and quantified in detail using OCT-A. This area seems particularly relevant not only in diabetes but also in many neurodegenerative disorders, including those involving the central nervous system. New studies are required, so a validated systematic approach to the evaluation of retinal microvasculature can be developed [12, 107].

The concept of the retina as a window to the brain has been explored in several studies that used ophthalmological examinations of the eye and retina to explore brain diseases. In particular, OCT-A has been, even if not completely validated, associated with neurological abnormalities such as cognitive impairment and Alzheimer's disease, multiple sclerosis, Parkinson's disease, Huntington's disease, amyotrophic lateral sclerosis, Wolfram syndrome, migraines, lesions of the visual pathway, and cerebral autosomal dominant arteriopathy with subcortical infarcts and leukoencephalopathy. It appears that OCT-A findings may contribute, in the future, to better understand the pathophysiology of these disorders [108]. Retinal microvasculature changes evaluated by OCT-A have also been associated with cardiovascular diseases, such as decrease in retinal vessel density and choroidal blood flow being associated with coronary stenosis. Eventually, OCT-A can be an efficient and noninvasive measurement method for detecting early stage of cardiovascular diseases, preventing long-term late stages [109, $110]$.

\section{The Role of Artificial Intelligence}

The quantitative OCT-A data offer a unique opportunity to develop informative tools based on artificial intelligence that will assist the clinicians in diagnosing, monitoring, and managing patients with diabetes. Machine learning techniques have already used OCT-A data to improve the diagnosis of DR-related findings such as image quality, retinal segmentation accuracy, and DR classification. However, the limited size of currently available databases and the variability of different OCT-A technologies are major challenging factors for deep learning-based technology [111].

\section{Conclusions}

OCT-A has revolutionized retinal imaging as it permits the identification of microvascular abnormalities even before the signs of DRD can be clinically detectable on fundus examination. This capacity of detecting early vascular impairment (i.e., low DCP perfusion density or 
decreased vessel response to flicker light) will be useful to design specific studies aimed at a specific component of DR such as determining the timing and onset of microvascular impairment or neurodegeneration in the development of DRD; to further characterize the phenotypes in early $\mathrm{DRD}$, and to test the neuroprotective and/or vasculotropic action of new drugs for treating DRD. In order to increase the value of OCT-A, there is an urgent need to establish a consensus nomenclature for OCT-A findings to standardize a clinical and scientific language. Further studies are warranted to evaluate and validate the use of OCT-A as an imaging biomarker in the management of DRD and future clinical trials.

\section{Acknowledgments}

This manuscript does not include any nonauthor contributions to acknowledge.

\section{Conflict of Interest Statement}

Stela Vujosevic reported being a member of Advisory Boards for Allergan, Alimera, Apellis, Bayer, Novartis, and Roche; none related to the content of this article. José G. Cunha-Vaz reported grants from Carl Zeiss Meditec and is consultant for Alimera Sciences, Allergan, Bayer, Gene Signal, Novartis, Pfizer, Precision Ocular Ltd., Roche, Sanofi-Aventis, Vifor Pharma, and Carl Zeiss Meditec. The funders had no role in the design or writing of the manuscript. João Figueira reported being a member of Advisory Boards for Alimera, Allergan, Bayer, Boehringer, and Novartis. The funders had no role in the design or writing of the manuscript. Anat
Löewenstein reported receiving consultant fees and grants from Allergan, Bayer, Notal Vision, Novartis, and Roche; consultant and advisory fees from KHB and Beyonics; and consultant fees from Syneos Health and WebMD. Edoardo Midena reported no conflict of interest. Mariacristina Parravano reported personal fees from Allergan, Bayer, Novartis, Sifi, and Zeiss. Peter Henry Scanlon reported consulting fees from Boehringer and Bayer and speaker fees from Novartis in the last 3 years. Rafael Simó reported receiving research support from NovoNordisk, Menarini, OM-VIFOR, Ferrer, and ABBOT; was an investigator in studies of Sanofi, NovoNordisk and Roche; and received consulting fees from NovoNordisk, MSD, Bayer, Novartis, AstraZeneca, Roche, OM-VIFOR, and Genesis Biomed. Tunde Peto reported receiving consultant/speaker's fees from Novartis, Bayer, Roche, Heidelberg, Roche, Optomed, and OPTOS; none related to the content of this paper. Cristina Hernández reported receiving research support from NovoNordisk, Menarini, OM-VIFOR, MSD, and Ferrer. Olga Simó-Servat reported receiving research support from NovoNordisk, Roche, OMVIFOR, Ferrer, and ABBOT. Ana R. Santos reported no conflict of interest. Maria H. Madeira reported no conflict of interest. Ines P. Marques reported no conflict of interest. Antonio C.-V. Martinho reported no conflict of interest. Dinah Zur reported receiving consulting fees from Allergan, Bayer, Novartis, and Roche. Recivall P. Salongcay reported no conflict of interest.

\section{Funding Sources}

This manuscript did not receive any funding.

\section{Author Contributions}

All authors significantly contributed to the writing and reviewing of the article as well as the final approval of the manuscript.

\section{References}

1 Early Treatment Diabetic Retinopathy Study Group. Grading diabetic retinopathy from stereoscopic color fundus photographs: an extension of the modified Airlie House classification: ETDRS report number 10. Ophthalmology. 1991;98(5 Suppl):786-806.

2 Early Treatment Diabetic Retinopathy Study Group. Photocoagulation treatment of proliferative diabetic retinopathy: the second report of diabetic retinopathy study findings. Ophthalmology. 1978;85(1):82-106.

3 Wilkinson CP, Ferris FL, Klein RE, Lee PP, Agardh CD, Davis M, et al. Proposed international clinical diabetic retinopathy and diabetic macular edema disease severity scales. Ophthalmology. 2003;110(9):1677-82.

4 Scanlon PH. The English National Screening Programme for diabetic retinopathy 20032016. Acta Diabetol. 2017;54(6):515-25.
5 Vujosevic S, Aldington SJ, Silva P, Hernández C, Scanlon P, Peto T, et al. Screening for diabetic retinopathy: new perspectives and challenges. Lancet Diabetes Endocrinol. 2020; 8(4):337-47.

6 Vujosevic S, Toma C, Villani E, Muraca A, Torti E, Florimbi G, et al. Diabetic macular edema with neuroretinal detachment: OCT and OCT-angiography biomarkers of treatment response to anti-VEGF and steroids. Acta Diabetol. 2020;57(3):287-96.

7 Couturier A, Mané V, Bonnin S, Erginay A, Massin P, Gaudric A, et al. Capillary plexus anomalies in diabetic retinopathy on optical coherence tomography angiography. Retina. 2015;35(11):2384-91.

8 Soares M, Neves C, Marques IP, Pires I, Schwartz C, Costa MÂ, et al. Comparison of diabetic retinopathy classification using fluorescein angiography and optical coherence tomography angiography. Br J Ophthalmol. 2017;101(1):62-8.
9 De Carlo TE, Zahid S, Bohm KJ, Chan RVP, Lim JI, Mieler WF. Simulating vascular leakage on optical coherence tomography angiography using an overlay technique with corresponding thickness maps. Br J Ophthalmol. 2020;104(4):514-7.

10 Farinha C, Santos T, Marques IP, Marques JP, Ribeiro L, Figueira J, et al. OCT-leakage mapping: a new automated method of OCT data analysis to identify and locate abnormal fluid in retinal edema. Ophthalmol Retina. 2017; 1(6):486-96.

11 Rodríguez FJ, Staurenghi G, Gale R. The role of OCT-A in retinal disease management. Graefes Arch Clin Exp Ophthalmol. 2018; 256(11):2019-26.

12 Vujosevic S, Muraca A, Gatti V, Masoero L, Brambilla M, Cannillo B, et al. Peripapillary microvascular and neural changes in diabetes mellitus: an oct-angiography study. Invest Ophthalmol Vis Sci. 2018;59(12):5074-81. 
13 Ashraf M, Sampani K, Clermont A, Abu-Qamar O, Rhee J, Silva PS, et al. Vascular density of deep, intermediate and superficial vascular plexuses are differentially affected by diabetic retinopathy severity. Invest Ophthalmol Vis Sci. 2020;61(10):53.

14 Akil H, Karst S, Heisler M, Etminan M, Navajas E, Maberley D. Application of optical coherence tomography angiography in diabetic retinopathy: a comprehensive review. Can J Ophthalmol. 2019;54(5):519-28.

15 Vujosevic S, Muraca A, Alkabes M, Villani E, Cavarzeran F, Rossetti L, et al. Early microvascular and neural changes in patients with type 1 and type 2 diabetes mellitus without clinical signs of diabetic retinopathy. Retina. 2019;39(3):435-45.

16 Potsaid B, Baumann B, Huang D, Barry S, Cable AE, Schuman JS, et al. Ultrahigh speed $1050 \mathrm{~nm}$ swept source/Fourier domain OCT retinal and anterior segment imaging at 100,000 to 400,000 axial scans per second. Opt Express. 2010;18(19):20029.

17 De Vitis LA, Benatti L, Tomasso L, Baldin G, Carnevali A, Querques L, et al. Comparison of the performance of two different spectral-domain optical coherence tomography angiography devices in clinical practice. Ophthalmic Res. 2016;56(3):155-62.

18 Cole ED, Ferrara D, Novais EA, Louzada RN, Waheed NK. Clinical trial endpoints for optical coherence tomography angiography in neovascular age-related macular degeneration. Retina. 2016;36 Suppl 1:S83-92.

19 Chua J, Sim R, Tan B, Wong D, Yao X, Liu X, et al. Optical coherence tomography angiography in diabetes and diabetic retinopathy. $J$ Clin Med. 2020;9(6):1723.

20 Spaide RF. Volume-rendered optical coherence tomography of diabetic retinopathy pilot study. Am J Ophthalmol. 2015;160(6):120010.

21 Dimitrova G, Chihara E, Takahashi H, Amano H, Okazaki K. Author response: quantitative retinal optical coherence tomography angiography in patients with diabetes without diabetic retinopathy. Invest Ophthalmol Vis Sci. 2017;58(1):1767-6.

22 Rosen RB, Romo JSA, Krawitz BD, Mo S, Fawzi AA, Linderman RE, et al. Earliest evidence of preclinical diabetic retinopathy revealed using Optical Coherence Tomography Angiography (OCTA) perfused capillary density. Am J Ophthalmol. 2019;203:103-15.

23 Zahid S, Dolz-Marco R, Freund KB, Balaratnasingam $\mathrm{C}$, Dansingani $\mathrm{K}$, Gilani $\mathrm{F}$, et al. Fractal dimensional analysis of optical coherence tomography angiography in eyes with diabetic retinopathy. Invest Ophthalmol Vis Sci. 2016;57(11):4940-7.

24 Borrelli E, Sadda SR, Uji A, Querques G. Pearls and pitfalls of optical coherence tomography angiography imaging: a review. Ophthalmol Ther. 2019;8(2):215-26.
25 Vujosevic S, Toma C, Villani E, Gatti V, Brambilla M, Muraca A, et al. Early detection of microvascular changes in patients with diabetes mellitus without and with diabetic retinopathy: comparison between different swept-source OCT-A instruments. J Diabetes Res. 2019;2019:2547216.

26 Choi J, Kwon J, Shin JW, Lee J, Lee S, Kook MS. Quantitative optical coherence tomography angiography of macular vascular structure and foveal avascular zone in glaucoma. PLoS One. 2017;12(9):e0184948.

27 Borrelli E, Balasubramanian S, Triolo G, Barboni P, Sadda SR, Sadun AA. Topographic macular microvascular changes and correlation with visual loss in chronic leber hereditary optic neuropathy. Am J Ophthalmol. 2018;192:217-28.

28 Spaide RF, Fujimoto JG, Waheed NK. Optical coherence tomography angiography. Retina. 2015;35(1):2161-2.

29 Spaide RF. Choriocapillaris flow features follow a power law distribution: implications for characterization and mechanisms of disease progression. Am J Ophthalmol. 2016;170:5867.

30 Dodo Y, Suzuma K, Ishihara K, Yoshitake S, Fujimoto M, Yoshitake T, et al. Clinical relevance of reduced decorrelation signals in the diabetic inner choroid on optical coherence tomography angiography. Sci Rep. 2017;7(1): 5227-11.

31 Tian M, Wolf S, Munk MR, Schaal KB. Evaluation of different Swept-Source optical coherence tomography angiography (SS-OCTA) slabs for the detection of features of diabetic retinopathy. Acta Ophthalmol. 2020;98(4): e416-20.

32 Kashani AH, Lee SY, Moshfeghi A, Durbin MK, Puliafito CA. Optical coherence tomography angiography of retinal venous occlusion. Retina. 2015 Nov;35(11):2323-31.

33 You QS, Chan JCH, Ng ALK, Choy BKN, Shih $\mathrm{KC}$, Cheung JJC, et al. Macular vessel density measured with optical coherence tomography angiography and its associations in a large population-based study. Invest Ophthalmol Vis Sci. 2019;60(14):4830-7.

34 Munk MR, Giannakaki-Zimmermann $\mathrm{H}$, Berger L, Huf W, Ebneter A, Wolf S, et al. OCT-angiography: a qualitative and quantitative comparison 4 OCT-A devices. PLoS One. 2017;12(5):e0177059.

35 Corvi F, Corradetti G, Parrulli S, Pace L, Staurenghi G, Sadda SR. Comparison and repeatability of high resolution and high speed scans from spectralis optical coherence tomography angiography. Transl Vis Sci Technol. 2020;9(10):29-10.

36 Rabiolo A, Gelormini F, Sacconi R, Cicinelli MV, Triolo G, Bettin P, et al. Comparison of methods to quantify macular and peripapillary vessel density in optical coherence tomography angiography. PLoS One. 2018; 13(10):e0205773-20.
37 Corvi F, Sadda SR, Staurenghi G, Pellegrini M. Thresholding strategies to measure vessel density by optical coherence tomography angiography. Can J Ophthalmol. 2020;55(4): $317-22$

38 Munk MR, Kashani AH, Tadayoni R, Korobelnik JF, Wolf S, Pichi F, et al. Standardization of OCT angiography nomenclature in retinal vascular diseases: first survey results. Ophtalmol Retina. 2021 Jan 1. Epub ahead of print.

39 Lu Y, Wang JC, Cui Y, Zhu Y, Zeng R, Lu ES, et al. A quantitative comparison of four optical coherence tomography angiography devices in healthy eyes. Graefe's Arch Clin Exp Ophthalmol. 2021 Jun 1;259(6):1493-1501.

40 Frank R, Hargreaves R. Clinical biomarkers in drug discovery and development. Nat Rev Drug Discov. 2003 Jul;2(7):566-80.

41 Ting DSW, Tan KA, Phua V, Tan GSW, Wong CW, Wong TY. Biomarkers of diabetic retinopathy. Curr Diab Rep. 2016 Dec;16(12): 125

42 Vujosevic S, Simó R. Local and systemic inflammatory biomarkers of diabetic retinopathy: an integrative approach. Invest Ophthalmol Vis Sci. 2017 May 1;58(6):BIO68-75.

43 Atchison E, Barkmeier A. The role of systemic risk factors in diabetic retinopathy. Curr Ophthalmol Rep. 2016;4(2):84-9.

44 Bhavsar AR, Emerson GG, Emerson MV, Browning DJ. Epidemiology of diabetic retinopathy. In: Browning DJ, editor. Diabetic retinopathy evidence-based management. New York: Springer; 2010.

45 Nunes S, Ribeiro L, Lobo C, Cunha-Vaz J. Three different phenotypes of mild nonproliferative diabetic retinopathy with different risks for development of clinically significant macular edema. Invest Ophthalmol Vis Sci. 2013;54(54):4595-604. 7

46 Hove MN, Kristensen JK, Lauritzen T, Bek T. The relationships between risk factors and the distribution of retinopathy lesions in type 2 diabetes. Acta Ophthalmol Scand. 2006;84(5): 619-23. Oct

47 Cunha-Vaz J, Ribeiro L, Lobo C. Phenotypes and biomarkers of diabetic retinopathy. Prog Retin Eye Res. 2014;41:90-111.

48 Martinho AC, Marques IP, Messias AL, Santos T, Madeira MH, Sousa DC, et al. Ocular and systemic risk markers for development of macular edema and proliferative retinopathy in type 2 diabetes: a 5-year longitudinal study. Diabetes Care. 2021;44(1):e12-14.

49 Tufail A, Rudisill C, Egan C, Kapetanakis VV, Salas-Vega S, Owen CG, et al. Automated diabetic retinopathy image assessment software: diagnostic accuracy and cost-effectiveness compared with human graders. Ophthalmology. 2017 Mar; 124(3):343-351.

50 Marques IP, Alves D, Santos T, Mendes L, Santos AR, Lobo C, et al. Multimodal imaging of the initial stages of diabetic retinopathy: different disease pathways in different patients. Diabetes. 2019;68(3):648-53. 
51 Bandello F, Cicinelli MV. 19th EURETINA congress keynote lecture: diabetic retinopathy today. Ophthalmologica. 2020;243(3): 163-71.

52 Marques IP, Alves D, Santos T, Mendes L, Lobo C, Santos AR, et al. Characterization of disease progression in the initial stages of retinopathy in type 2 diabetes: a 2-year longitudinal study. Invest Ophthalmol Vis Sci. 2020; 61(3):20.

53 FDA-NIH Biomarker Working Group. BEST (Biomarkers, EndpointS, and Other Tools) Resource [Internet]. 2016.

54 Marques IP, Madeira MH, Messias AL, Martinho ACV, Santos T, Sousa DC, et al. Different retinopathy phenotypes in type 2 diabetes predict retinopathy progression. Acta Diabetol. 2021;58:197-205.

55 Nesper PL, Roberts PK, Onishi AC, Chai H, Liu L, Jampol LM, et al. Quantifying microvascular abnormalities with increasing severity of diabetic retinopathy using optical coherence tomography angiography. Invest Ophthalmol Vis Sci. 2017 May; 58(6): BIO307-15.

56 Rodrigues TM, Marques JP, Soares M, Simão S, Melo P, Martins A, et al. Macular OCT-angiography parameters to predict the clinical stage of nonproliferative diabetic retinopathy: an exploratory analysis. Eye. 2019;33(8): $1240-7$.

57 Samara WA, Shahlaee A, Adam MK, Khan MA, Chiang A, Maguire JI, et al. Quantification of diabetic macular ischemia using optical coherence tomography angiography and its relationship with visual acuity. Ophthalmology. 2017;124(2):235-44.

58 Durbin MK, An L, Shemonski ND, Soares M, Santos T, Lopes M, et al. Quantification of retinal microvascular density in optical coherence tomographic angiography images in diabetic retinopathy. JAMA Ophthalmol. 2017; 135(4):370.

59 Ashraf M, Sampani K, Rageh A, Silva PS, Aiello LP, Sun JK. Interaction between the distribution of diabetic retinopathy lesions and the Association of Optical Coherence Tomography Angiography scans with diabetic retinopathy severity. JAMA Ophthalmol. 2020;138:1291.

60 Cui Y, Zhu Y, Wang JC, Lu Y, Zeng R, Katz R, et al. Comparison of widefield swept-source optical coherence tomography angiography with ultra-widefield colour fundus photography and fluorescein angiography for detection of lesions in diabetic retinopathy. $\mathrm{Br} \mathrm{J}$ Ophthalmol. 2020:316245.

61 Santos T, Lewis W, Santos AR, Marques IP, Kubach S, Mendes LG, et al. Swept source OCTA quantification of capillary closure predicts ETDRS severity staging of NPDR. Br J Ophthalmol. 2020. Epub ahead of print.

62 Tan B, Chua J, Lin E, Cheng J, Gan A, Yao X, et al. Quantitative microvascular analysis with wide-field optical coherence tomography angiography in eyes with diabetic retinopathy. JAMA Netw open. 2020;3(1):e1919469.
63 Chua J, Schmetterer L. Letter to the Editor on "macular OCT-angiography parameters to predict the clinical stage of nonproliferative diabetic retinopathy: an exploratory analysis". Eye. Published online. 2020 Dec;34(12): 2341-2.

64 Marques IP, Madeira MH, Messias AL, Santos T, Martinho AC-V, Figueira J, et al. Retinopathy phenotypes in type 2 diabetes with different risks for macular edema and proliferative retinopathy. J Clin Med. 2020;9(5):1433.

65 Pires I, Santos AR, Nunes S, Lobo C, CunhaVaz J. Subclinical macular edema as a predictor of progression to clinically significant macular edema in type 2 diabetes. Ophthalmologica. 2013;230(4):201

66 Scarinci F, Nesper PL, Fawzi AA. Deep retinal capillary nonperfusion is associated with photoreceptor disruption in diabetic macular ischemia. Am J Ophthalmol. 2016 Aug; 168: 129-38.

67 Califf RM. Biomarker definitions and their applications. Exp Biol Med. 2018;243(3):213221.

68 Early Treatment Diabetic Retinopathy Study Research Group. Grading diabetic retinopathy from stereoscopic color fundus photographs: an extension of the modified airlie house classification: ETDRS report number 10. Ophthalmology. 2020. 127(4S, S99):S9: S99-S119.

69 Hwang TS, Jia Y, Gao SS, Bailey ST, Lauer AK, Flaxel CJ, et al. Optical coherence tomography angiography features of diabetic retinopathy. Retina. 2015;35(11):2371-6.

70 Kowluru RA, Chan P-S. Capillary dropout in diabetic retinopathy. In: Duh E, editor. Diabetic retinopathy. Totowa: Humana Press; 2008. p. 265-82.

71 Parravano M, Costanzo E, Querques G. Profile of non-responder and late responder patients treated for diabetic macular edema: systemic and ocular factors. Acta Diabetol. 2020; 57(8):911-21.

72 Lee J, Moon BG, Cho AR, Yoon YH. Optical coherence tomography angiography of DME and its association with anti-VEGF treatment response. Ophthalmology. 2016;123(11): 2368-75.

73 Hsieh YT, Alam MN, Le D, Hsiao CC, Yang $\mathrm{CH}$, Chao DL, et al. OCT angiography biomarkers for predicting visual outcomes after ranibizumab treatment for diabetic macular edema. Ophthalmol Retina. 2019;3(1):82634.

74 Parravano M, De Geronimo D, Scarinci F, Querques L, Virgili G, Simonett JM, et al. Diabetic microaneurysms internal reflectivity on spectral-domain optical coherence tomography and optical coherence tomography angiography detection. Am J Ophthalmol. 2017; 179:90-6.

75 Markan A, Agarwal A, Arora A, Bazgain K, Rana V, Gupta V. Novel imaging biomarkers in diabetic retinopathy and diabetic macular edema. Ther Adv Ophthalmol. 2020;12(6): 2515841420950513.
76 Busch C, Wakabayashi T, Sato T, Fukushima Y, Hara C, Shiraki N, et al. Retinal microvasculature and visual acuity after intravitreal aflibercept in diabetic macular edema: an optical coherence tomography angiography study. Sci Rep. 2019;9(1):1561-9.

77 Sorour OA, Sabrosa AS, Yasin Alibhai A, Arya M, Ishibazawa A, Witkin AJ, et al. Optical coherence tomography angiography analysis of macular vessel density before and after anti-VEGF therapy in eyes with diabetic retinopathy. Int Ophthalmol. 2019;39(10):236171.

78 Couturier A, Rey PA, Erginay A, Lavia C, Bonnin S, Dupas B, et al. Widefield OCT-angiography and fluorescein angiography assessments of nonperfusion in diabetic retinopathy and edema treated with anti-vascular endothelial growth factor. Ophthalmology. 2019;126(12):1685-94.

79 Kim YJ, Yeo JH, Son G, Kang H, Sung YS, Lee JY, et al. Efficacy of intravitreal AFlibercept injection for Improvement of retinal Nonperfusion in diabeTic retinopathY (AFFINITY study). BMJ Open Diabetes Res Care. 2020; 8(1):1-8.

80 Balaratnasingam C, Inoue M, Ahn S, McCann J, Dhrami-Gavazi E, Yannuzzi LA, et al. Visual acuity is correlated with the area of the foveal avascular zone in diabetic retinopathy and retinal vein occlusion. Ophthalmology. 2016;123(11):2352-67.

81 Toto L, D’Aloisio R, Chiarelli AM, Di Antonio L, Evangelista F, D'Onofrio G, et al. A custom-made semiautomatic analysis of retinal nonperfusion areas after dexamethasone for diabetic macular edema. Transl Vis Sci Technol. 2020;9(7):13.

82 Scarinci F, Picconi F, Giorno P, Boccassini B, De Geronimo D, Varano M, et al. Deep capillary plexus impairment in patients with type 1 diabetes mellitus with no signs of diabetic retinopathy revealed using optical coherence tomography angiography. Acta Ophthalmol. 2018;96(2):e264-5.

83 Sun Z, Tang F, Wong R, Lok J, Szeto SKH, Chan JCK, et al. OCT angiography metrics predict progression of diabetic retinopathy and development of diabetic macular edema: a prospective study. Ophthalmology. 2019; 126(12):1675-84.

84 Scarinci F, Picconi F, Virgili G, Varano M, Giorno P, Frontoni S, et al. Microvascular impairment as a biomarker of diabetic retinopathy progression in the long-term follow up in type 1 diabetes. Sci Rep. 2020;10(1):18266-10.

85 Dorner GT, Garhöfer G, Huemer KH, Riva CE, Wolzt M, Schmetterer L. Hyperglycemia affects flicker-induced vasodilation in the retina of healthy subjects. Vision Res. 2003; 43(13):1495-500.

86 Kwan CC, Lee HE, Schwartz G, Fawzi AA Acute hyperglycemia reverses neurovascular coupling during dark to light adaptation in healthy subjects on optical coherence tomography angiography. Invest Ophthalmol Vis Sci. 2020;61(4):38. 
87 Lee WH, Park JH, Won Y, Lee MW, Shin YI, Jo YJ, et al. Retinal microvascular change in hypertension as measured by optical coherence tomography angiography. Sci Rep. 2019; 9(1):156-7.

88 Chua J, Chin CWL, Hong J, Chee ML, Le TT, Ting DSW, et al. Impact of hypertension on retinal capillary microvasculature using optical coherence tomographic angiography. J Hypertens. 2019;37(3):572-80.

89 Hannappe MA, Arnould L, Méloux A, Mouhat B, Bichat F, Zeller M, et al. Vascular density with optical coherence tomography angiography and systemic biomarkers in low and high cardiovascular risk patients. Sci Rep. 2020;10(1):16718.

90 Wong TY, Liew G, Tapp RJ, Schmidt MI, Wang JJ, Mitchell P, et al. The relationship of fasting glucose to retinopathy: re-visiting a key criterion used to diagnose diabetes. Lancet. 2008;371:736-43.

91 Zhang J, Qiao Y, Sarabi MS, Khansari MM, Gahm JK, Kashani AH, et al. 3D shape modeling and analysis of retinal microvasculature in OCT-angiography images. IEEE Trans Med Imaging. 2020;39(5):1335-46.

92 Prescott JW. Quantitative imaging biomarkers: the application of advanced image processing and analysis to clinical and preclinical decision making. J Digit Imaging. 2013;26(1):97-108.

93 Lee J, Rosen R. Optical coherence tomography angiography in diabetes. Curr Diab Rep. 2016;16(12): 123 .

94 Ghasemi Falavarjani K, Al-Sheikh M, Akil H, Sadda SR. Image artefacts in swept-source optical coherence tomography angiography. $\mathrm{Br}$ J Ophthalmol. 2017;101(5):564-8.

95 Zhang M, Hwang TS, Dongye C, Wilson DJ, Huang D, Jia Y. Automated quantification of nonperfusion in three retinal plexuses using projection-resolved optical coherence tomography angiography in diabetic retinopathy. Invest Ophthalmol Vis Sci. 2016;57(13): 5101-6.
96 Hwang TS, Zhang M, Bhavsar K, Zhang X, Campbell JP, Lin P, et al. Visualization of 3 distinct retinal plexuses by projection-resolved optical coherence tomography angiography in diabetic retinopathy. JAMA Ophthalmol. 2016;134(12):1411-9.

97 Jia Y, Bailey ST, Hwang TS, McClintic SM, Gao SS, Pennesi ME, et al. Quantitative optical coherence tomography angiography of vascular abnormalities in the living human eye. Proc Natl Acad Sci U S A. 2015;112: E2395-402.

98 Lauermann JL, Eter N, Alten F. Optical coherence tomography angiography offers new insights into choriocapillaris perfusion. Ophthalmologica. 2018;239:74-84.

99 Uji A, Balasubramanian S, Lei J, Baghdasaryan E, Al-Sheikh M, Sadda SR. Choriocapillaris imaging using multiple en face optical coherence tomography angiography image averaging. JAMA Ophthalmol. 2017;135:1197-204.

100 Chu Z, Zhang Q, Gregori G, Rosenfeld PJ, Wang RK. Guidelines for imaging the choriocapillaris using OCT angiography. Am J Ophthalmol. 2021 Feb;222:92-101.

101 Lupidi M, Cerquaglia A, Gujar R, Fruttini D, Cardillo Piccolino F, Fiore T, et al. Functional correlation between choroidal and retinal vascularity in low-grade diabetic retinopathy. Acta Diabetol. 2020 Aug;57(8):983-90.

102 Dai Y, Zhou H, Zhang Q, Chu Z, Olmos de Koo LC, Chao JR, et al. Quantitative assessment of choriocapillaris flow deficits in diabetic retinopathy: a swept-source optical coherence tomography angiography study. PLoS One. 2020 Dec 11;15(12):e0243830.

103 Sorour O, Arya M, Waheed N. New findings and challenges in OCT angiography for diabetic retinopathy. Ann Eye Sci. 2018;3(8): 44.
104 Parrulli S, Corvi F, Cozzi M, Monteduro D, Zicarelli F, Staurenghi G. Microaneurysms visualisation using five different optical coherence tomography angiography devices compared to fluorescein angiography. $\mathrm{Br}$ Ophthalmol. 2021;105(4).

105 Lujan BJ, Calhoun CT, Glassman AR, Googe JM, Jampol LM, Melia M, et al. Optical coherence tomography angiography quality across three multicenter clinical studies of diabetic retinopathy. Transl Vis Sci Technol. 2021;10(3):2.

106 Greig EC, Duker JS, Waheed NK. A practical guide to optical coherence tomography angiography interpretation. Int J Retina Vitreous. 2020;6(1):55.

107 Frizziero L, Parrozzani R, Londei D, Pilotto E, Midena E. Quantification of vascular and neuronal changes in the peripapillary retinal area secondary to diabetic retinopathy. $\mathrm{Br}$ ) Ophthalmol. 2020:1-7.

108 Tsokolas G, Tsaousis KT, Diakonis VF, Matsou A, Tyradellis S. Optical coherence tomography angiography in neurodegenerative diseases: a review. Eye Brain. 2020;12 73-87.

109 Wang J, Jiang J, Shang Y, Qian YW, Zhang JF, Wang ZL. Retinal and choroidal vascular changes in coronary heart disease : an optical coherence tomography angiography study. Biomed Opt Express. 2019;10(4): 1532-44.

110 Courtie E, Veenith T, Logan A, Denniston AK, Blanch RJ. Retinal blood flow in critical illness and systemic disease : a review. Ann Intensive Care. 2020;10:152.

111 Yao X, Alam MN, Le D, Toslak D. Quantitative optical coherence tomography angiography: A review. Exp Biol Med. 2020;245: 301-12. 